\title{
Measure of synonymous codon usage diversity among genes in bacteria
}

\author{
Haruo Suzuki1,2, Rintaro Saito*1 and Masaru Tomita ${ }^{1}$
}

Address: ${ }^{1}$ Institute for Advanced Biosciences, Keio University, Tsuruoka, Yamagata, 997-0017, Japan and ${ }^{2}$ Systems Biology Program, Graduate School of Media and Governance, Keio University, Fujisawa, Kanagawa, 252-8520, Japan

Email: Haruo Suzuki - haruo@g-language.org; Rintaro Saito* - rsaito@sfc.keio.ac.jp; Masaru Tomita - mt@sfc.keio.ac.jp

* Corresponding author

Published: I June 2009

BMC Bioinformatics 2009, 10:167 doi:10.1186/1471-2105-10-167
Received: 27 October 2008

Accepted: I June 2009

This article is available from: http://www.biomedcentral.com/I47I-2/05//0/167

(C) 2009 Suzuki et al; licensee BioMed Central Ltd.

This is an Open Access article distributed under the terms of the Creative Commons Attribution License (http://creativecommons.org/licenses/by/2.0), which permits unrestricted use, distribution, and reproduction in any medium, provided the original work is properly cited.

\begin{abstract}
Background: In many bacteria, intragenomic diversity in synonymous codon usage among genes has been reported. However, no quantitative attempt has been made to compare the diversity levels among different genomes. Here, we introduce a mean dissimilarity-based index (Dmean) for quantifying the level of diversity in synonymous codon usage among all genes within a genome.

Results: The application of Dmean to 268 bacterial genomes shows that in bacteria with extremely biased genomic $\mathrm{G}+\mathrm{C}$ compositions there is little diversity in synonymous codon usage among genes. Furthermore, our findings contradict previous reports. For example, a low level of diversity in codon usage among genes has been reported for Helicobacter pylori, but based on Dmean, the diversity level of this species is higher than those of more than half of bacteria tested here. The discrepancies between our findings and previous reports are probably due to differences in the methods used for measuring codon usage diversity.

Conclusion: We recommend that Dmean be used to measure the diversity level of codon usage among genes. This measure can be applied to other compositional features such as amino acid usage and dinucleotide relative abundance as a genomic signature.
\end{abstract}

\section{Background}

Most amino acids can be encoded by more than one codon (i.e., a triplet of nucleotides); such codons are described as being synonymous, and usually differ by one nucleotide in the third position. In most bacteria, alternative synonymous codons are not used with equal frequencies. Grantham et al. [1] showed that genes from same species often show similar patterns of codon usage, and proposed the 'genome hypothesis' that there exists a species-specific pattern of codon usage. Then, it was shown that in many organisms there are also considerable differences in codon usage among genes within a genome [2]. Previous analyses of codon usage diversity in bacteria have mostly focused on individual genomes, with no quantitative attempt to compare the diversity levels among different genomes. For comparative genomic analysis, it is desirable to quantify the level of codon usage diversity among genes in such a way that the estimates could be compared among genomes.

Different factors have been proposed to explain the preferential usage of a subset of synonymous codons, including biased mutation pressure (genome-wide mutational bias toward $\mathrm{G} / \mathrm{C}$ or $\mathrm{A} / \mathrm{T}$ ) [3], difference in mutational bias between the leading and lagging strands of DNA replication (strand-specific mutational bias) $[4,5]$, and natural 
selection for optimizing translation process (translational selection) [6]. Although the genome-wide mutational bias should act on the entire genome, the extent is stronger for the third positions of codons since the first two positions of codons are constrained by protein-coding requirements [7]. Thus, the mutational bias could be the cause of the preferential usage of either G/C- or A/T-ending codons. The strand-specific mutational bias could be the cause of the preferential usage of $\mathrm{G} / \mathrm{T}$ - and $\mathrm{C} / \mathrm{A}$-ending codons in the leading and lagging strands, respectively $[8,9]$. The translational selection should act mainly on genes expressed at high levels in fast-growing bacteria [6]. The selection could be the cause of the preferential usage of translationally optimal codons, which are best recognized by the most abundant tRNA species in the cell $[10,11]$. It was reported that correlations of codon usage bias with gene expression level [6] and $\mathrm{G}+\mathrm{C}$ content bias [12] are not ubiquitous. Thus, codon usage diversity within any genome could be the result of a balance among different evolutionary forces, and their relative contributions vary among different genomes.

Different methods have been used to examine codon usage diversity among genes [2,13-15]. Univariate statistics such as the 'effective number of codons' (ENC) [16] and $\mathrm{G}+\mathrm{C}$ content at the third codon position (GC3) have been used to summarize codon usage of a gene. Representation of codon usage of a gene by a single statistic is essentially a reduction in information. GC3 estimates codon usage bias only toward either G/C- or A/T-ending codons. ENC estimates the degree of codon usage bias, but does not provide information about the types of preferred codons; thus two genes can exhibit same ENC values but prefer totally different codons. Multivariate analysis methods such as correspondence analysis (CA) have been used to construct axes accounting for the largest fractions of the total variation in codon usage among genes. In most genomes, the first two or three CA axes explain rather small amount of the total variation [13-15]. Carbone et al. [17] used the codon adaptation index (CAI) [18] as a universal measure of dominating codon usage bias. CA axis scores and CAI values derived from independent analyses cannot be compared. These limitations of previously used methods motivated us to consider alternative approach for measuring codon usage diversity.

In the present study, we introduce a mean dissimilaritybased index (Dmean) for quantifying the level of diversity in synonymous codon usage among all genes within a genome. This index has been used to measure bacterial diversity $[19,20]$. The Dmean values can be used to rank different genomes with respect to the overall codon usage diversity. The application of Dmean to 268 bacterial genomes demonstrates that in bacteria with extremely biased genomic $\mathrm{G}+\mathrm{C}$ compositions there is little diversity in synonymous codon usage among genes. Furthermore, our findings contradict the results of previous studies, and the reasons for the discrepancies are discussed.

\section{Results}

\section{Synonymous codon usage diversity (Dmean)}

To quantify the dissimilarity in synonymous codon usage between two genes, we calculated Pearson correlation distance $(D)$. Figure 1 shows histograms generated by all pairwise $D$ values among all protein-coding genes within each of two genomes: Borrelia burgdorferi B31 and Treponema pallidum Nichols as examples. In these two spirochaetes, there is a clear base composition skew between leading and lagging strands of replication [5]. The $D$ values for B. burgdorferi exhibited a bimodal distribution with a left peak corresponding to within-strand dissimilarities and a right peak corresponding to between-strand dissimilarities (Figure 1A), whereas those for T. pallidum exhibited a monomodal distribution (Figure 1B). As a whole, the $D$ values tended to be smaller in $B$. burgdorferi than in T. pallidum.

To quantify the level of diversity in synonymous codon usage among all genes within a genome, we calculated the mean distance (Dmean) between all pairs of genes. The Dmean value for $B$. burgdorferi (0.27) was smaller than that for T. pallidum (0.64), indicating that the diversity level was lower in $B$. burgdorferi than in $T$. pallidum. Among the 268 bacterial genomes tested here, the Dmean values ranged from 0.09 to 0.70 , with the median of 0.36 [see Additional file 1]. When focusing on previously studied genomes [13,14,21], the Dmean values for Bacillus subtilis 168 (0.60), Escherichia coli K12 MG1655 (0.47), Helicobacter pylori 26695 (0.38), and Haemophilus influenzae Rd KW20 (0.37) were above the median, while those for Pseudomonas aeruginosa PAO1 (0.15) and Streptomyces avermitilis MA-4680 (0.14) were below the median. Thus, Dmean values varied widely among bacteria.

\section{Relationship of Dmean with genomic features}

To investigate whether the level of intragenomic diversity in synonymous codon usage among genes is related to genomic features, we analyzed correlations of Dmean with genomic $\mathrm{G}+\mathrm{C}$ composition, replication strand skew, and tRNA gene number. The genomic $\mathrm{G}+\mathrm{C}$ content (\%GC) was expressed as $100 \times(\mathrm{G}+\mathrm{C}) /(\mathrm{A}+\mathrm{T}+\mathrm{G}+\mathrm{C})$. The strength of replication strand skew was quantified by the GC skew index (GCSI), which uses the power spectrum of Fourier transform of the graph of GC skew [the quantity $(\mathrm{C}-\mathrm{G}) /(\mathrm{C}+\mathrm{G})]$ and the Euclidean distance between the peaks [22]. Among the 268 bacterial genomes tested here, $\% \mathrm{GC}, \mathrm{GCSI}$, and tRNA gene numbers varied from 22.5 to $74.9,0.005$ to 0.715 , and 27 to 145 , respectively [see Additional file 1]. 

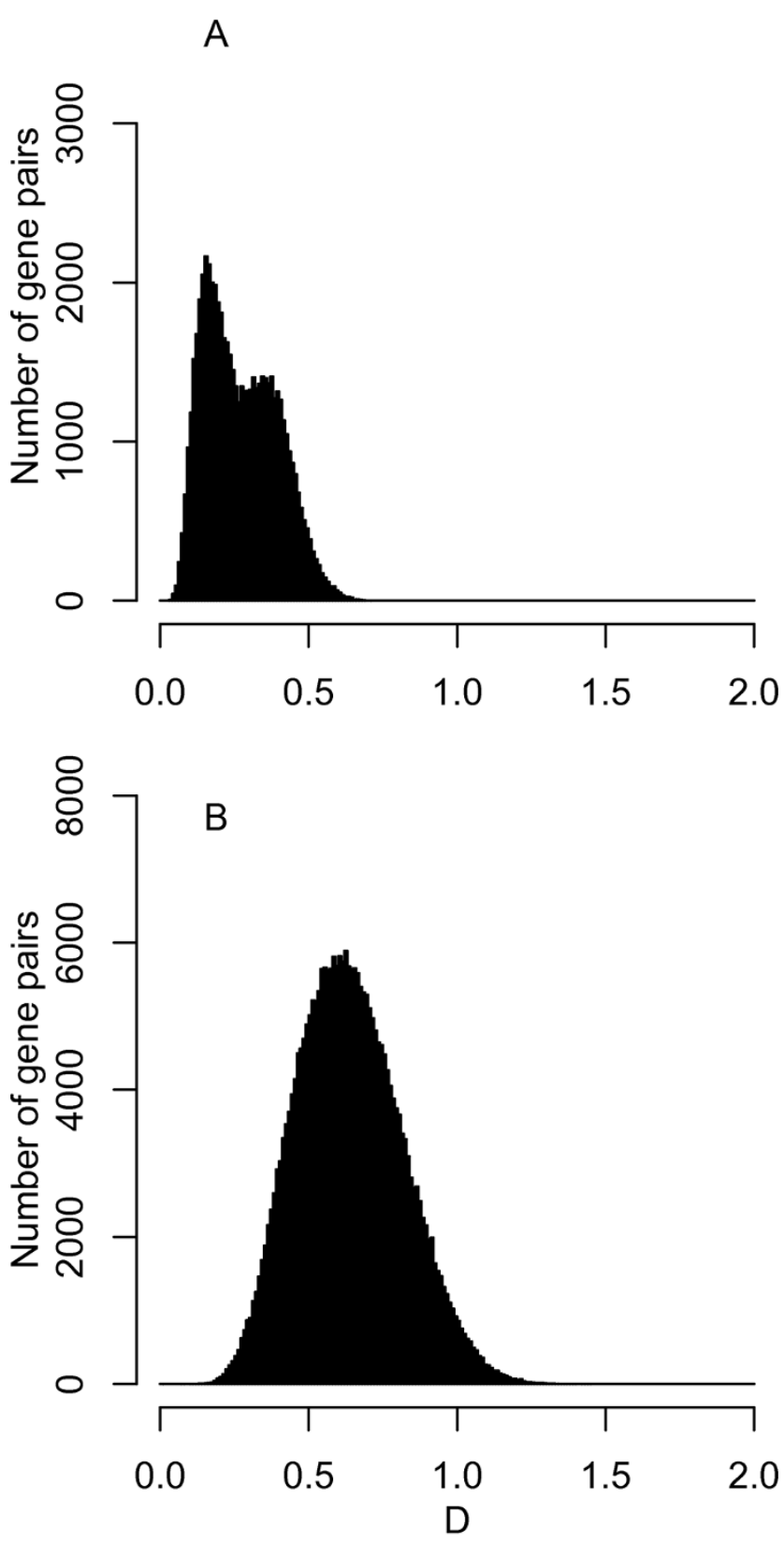

Figure I

Synonymous codon usage dissimilarity between genes. Histograms showing the distribution of Pearson correlation distance $(D)$ values between all pairs of all proteincoding genes within each of two genomes: Borrelia burgdorferi B3 I (A) and Treponema pallidum Nichols (B).
Figure 2 shows scatter plots of the Dmean values plotted against \%GC, GCSI, and tRNA gene numbers for 268 bacterial genomes. The Dmean values were nonlinearly correlated with \%GC (Figure 2A). The highest Dmean value (0.70) was found in Prochlorococcus marinus MIT 9303 $(\% \mathrm{GC}=50.0)$. The Dmean values tended to be low in bacteria with extremely biased genomic $\mathrm{G}+\mathrm{C}$ compositions (either $\mathrm{G}+\mathrm{C}$ - or $\mathrm{A}+\mathrm{T}-\mathrm{rich}$ ). Although these two types of genomes prefer different codons (either G/C- or A/T-ending codons), they can exhibit same Dmean values. For example, Wigglesworthia glossinidia (endosymbiont of Glossina brevipalpis) and Sorangium cellulosum 'So ce 56' had very different \%GC (22.5 and 71.4, respectively) but exhibited same Dmean values (0.13). In contrast to \%GC (Figure 2A), GCSI (Figure 2B) and tRNA gene numbers (Figure 2C) were not clearly correlated with the Dmean values.

\section{Comparison of Dmean with previous methods}

In previous studies, the extent of dispersal, e.g., range or standard deviation (SD), of univariate statistics such as the 'effective number of codons' (ENC) and $\mathrm{G}+\mathrm{C}$ content at the third codon position (GC3) has been used to measure codon usage diversity among genes $[13,14,21]$. We compared Dmean with SD of ENC and SD of GC3, designated as SD-ENC and SD-GC3, respectively.

Figures 3A and 3B show scatter plots of SD-ENC and SDGC3 plotted against Dmean for the 268 bacterial genomes. The correlations of Dmean with SD-ENC (Figure 3A) and SD-GC3 (Figure 3B) were unclear. The square of Pearson's product moment correlation coefficient of Dmean with SD-ENC and SD-GC3 indicates that only $0.01 \%$ and $13.0 \%$ of the variance in Dmean was explained by the variance in SD-ENC and SD-GC3, respectively.

Figures 3C and 3D show scatter plots of SD-ENC and SDGC3 plotted against \%GC for the 268 bacterial genomes. The nonlinear correlation with \%GC was clearer when using Dmean (Figure 2A) than when using SD-ENC (Figure 3C) and SD-GC3 (Figure 3D).

\section{Discussion}

In many bacteria, intragenomic diversity in codon usage among genes has been reported $[2,11]$. However, no quantitative attempt has been made to compare the diversity levels among different genomes. Here, we used a mean distance (Dmean) between all pairs of genes as a diversity index [20].

Different factors could contribute to codon usage diversity, including $\mathrm{G}+\mathrm{C}$ composition, strand-specific mutational bias, and translational selection [23]. First, in bacteria with extremely biased genomic $\mathrm{G}+\mathrm{C}$ compositions, synonymous codon usage could be dominated by 

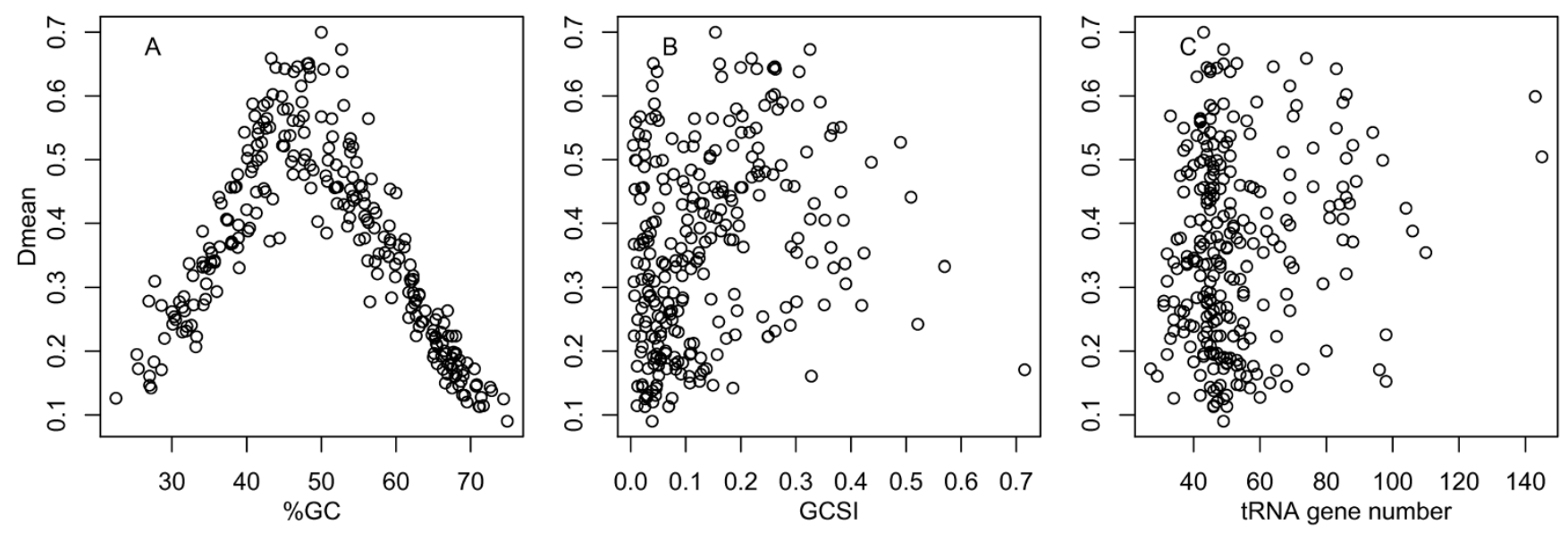

Figure 2

Relationship of Dmean with genomic features. Scatter plots of Dmean plotted against \%GC (A), GCSI (B), and tRNA gene number (C) for 268 bacterial genomes.

strong genome-wide mutational biases [3,7]. The nonlinear correlation between Dmean and \%GC (Figure 2A) suggests that the biased mutational pressures homogenize codon usage throughout the genome. Such 'genome amelioration' is postulated to detect horizontally transferred genes based on unusual codon usage $[24,25]$. G+C composition could reflect not only mutational biases but also other factors such as chromosomal position [26], oxygen requirement [27], and energy cost and availability of nucleotides [28].

Second, in bacteria with clear base composition skews along the genome, synonymous codon usage could be subject to strong strand-specific mutational biases $[8,9]$. In B. burgdorferi, the Dmean values for genes on the leading and lagging strands of replication were 0.19 and 0.20 , respectively. The corresponding values in T. pallidum were 0.54 and 0.62 . In these two spirochaetes, the Dmean values for all genes ( 0.27 and 0.64 , respectively) were larger than those for genes on each of the two replication strands, indicating that replication strand skew contributes to the overall codon usage diversity. The weak correlation between Dmean and GCSI (Figure 2B) suggests that the strand-specific mutational biases contribute less to the overall codon usage diversity than the evolutionary forces that determine $\mathrm{G}+\mathrm{C}$ composition.

Third, in bacteria with many tRNA genes, synonymous codon usage could be subject to strong translational selection [6]. The negligible correlation between Dmean and tRNA gene numbers (Figure 2C) suggests that translational selection contributes little to the overall codon usage diversity. A possible explanation for this observation is that the number of highly expressed genes on which translational selection has been effective is a very small fraction of the genome.

The use of Dmean led to the conclusions contrary to those drawn from previous studies. For example, a low level of heterogeneity in codon usage among genes has been reported for $H$. pylori genome in two independent analyses $[14,17]$. However, more than half of bacteria tested here had lower Dmean values than the Dmean value of $H$. pylori (0.38), indicating a moderate level of synonymous codon usage diversity in that genome. Also, clear and considerable heterogeneity in codon usage among genes has been reported for $P$. aeruginosa [13] and S. avermitilis [21], but their Dmean values were very small $(0.15$ and 0.14 , respectively), indicating a low level of synonymous codon usage diversity in these genomes. Previously used measures such as ENC (Figure 3A) and GC3 (Figure 3B) explain only a small percentage of the total variation in Dmean. Furthermore, the nonlinear correlation between codon usage diversity and \%GC was unclear when using ENC (Figure 3C) and GC3 (Figure 3D) instead of Dmean (Figure 2A). Thus, the discrepancies between our findings and previous reports are probably due to differences in the methods used for measuring codon usage diversity.

\section{Conclusion}

We recommend that Dmean be used to measure the diversity level of codon usage among genes. This measure can be applied to other compositional features such as amino acid usage $[29,30]$ and dinucleotide relative abundance as a genomic signature [31,32], and any groups of genes such as those encoding ribosomal proteins and aminoacyltRNA synthetases. The combined use of Dmean and complementary methods [6,17,33-35] will improve our understanding of compositional diversity among genes. 

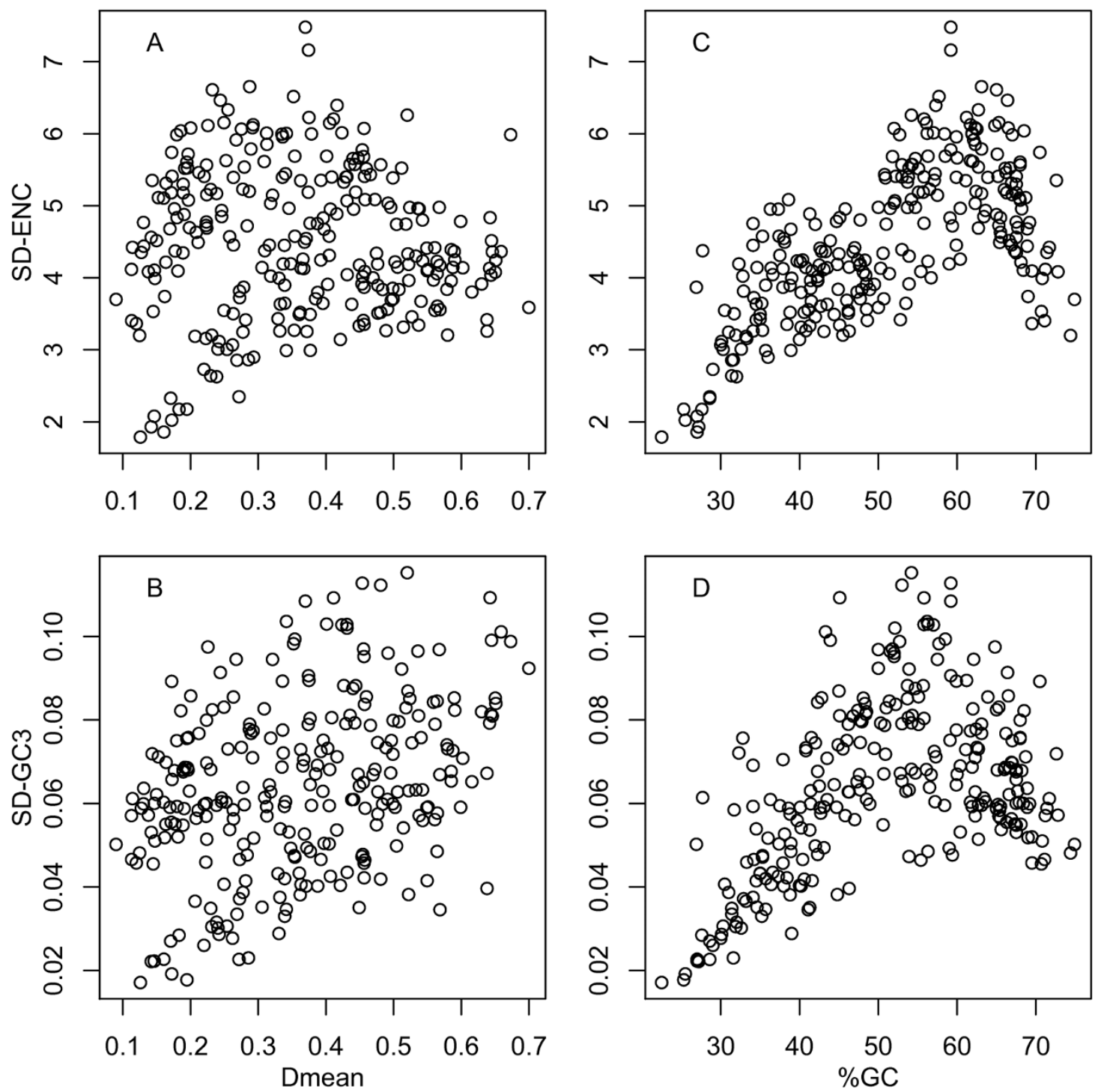

Figure 3

Comparison of Dmean with previous methods. Scatter plots of SD-ENC and SD-GC3 against Dmean (A and B) and \%GC (C and D) for 268 bacterial genomes.

\section{Methods}

\section{Softwares}

All analyses were implemented using the G-language Genome Analysis Environment version 1.8.3 [36,37] and the statistical software $\mathrm{R}$ version 2.6.1 [38].

\section{Sequences}

Complete genome sequences of bacteria in GenBank format [39] were retrieved from the NCBI [40] FTP site. For each genus, only one representative strain was selected. The final data set included 268 different genomes [see Additional file 1]. Protein coding sequences containing letters other than $A, C, G$, or $T$, and those containing amino acids with residues less than their degree of codon degeneracy were discarded. From each coding sequence, methionine, tryptophan, and stop codons were excluded.

Representation of synonymous codon usage of a gene Synonymous codon usage of a coding sequence was represented by a vector, which consists of 59 variables (codons). The value of the $c$ th codon for the ath amino acid $\left(x_{a c}\right)$ is defined as the ratio of the number of occurrences of a codon to the number of occurrences of the most abundant codon for the same amino acid [18]. 


$$
x_{a c}=\frac{n_{a c}}{\max \left(n_{a c}\right)}
$$

where $n_{a c}$ is the number of occurrences of $c$ th codon for the ath amino acid, and $\max \left(n_{a c}\right)$ is the number of occurrences of the most frequently used synonymous codon for the ath amino acid. The $x_{a c}$ value is independent of three biases (i.e., gene length, amino acid composition, and codon degeneracy) which can mask effects of synonymous codon usage [35].

\section{Measure of synonymous codon usage diversity among genes}

To quantify the dissimilarity in synonymous codon usage between two genes, we calculated Pearson correlation distance $(D)$, defined as one minus Pearson's product moment correlation coefficient. Let $X_{i}$ and $X_{j}$ be the vectors consisting of $59 x_{a c}$ values for the $i$ th and $j$ th genes, respectively. The $D$ value between the $i$ th and $j$ th genes $\left(D_{i j}\right)$ was calculated as:

$$
D_{i j}=1-\operatorname{cor}\left(X_{i}, X_{j}\right)
$$

where $\operatorname{cor}\left(X_{i}, X_{j}\right)$ is the correlation coefficient of $X_{i}$ and $X_{j}$. The correlation coefficient can vary from -1 (perfect negative correlation) through 0 (no correlation) to +1 (perfect positive correlation); thus the $D$ value can vary from 0 (minimum dissimilarity) to 2 (maximum dissimilarity).

To quantify the level of diversity in synonymous codon usage among all genes, we calculated the mean distance (Dmean) between all pairs of genes [20].

$$
\text { Dmean }=\frac{\sum D_{i j}}{N(N-1) / 2}
$$

where $N$ is the total number of genes. Dmean can reach the minimum value of 0 when all genes prefer same synonymous codons for all amino acids.

\section{Abbreviations}

A: adenine; T: thymine; G: guanine; C: cytosine; D: Pearson correlation distance; Dmean: mean distance between all pairs of genes as a diversity index; \%GC: genomic $\mathrm{G}+\mathrm{C}$ content; GCSI: GC skew index; SD-ENC: standard deviation of the effective number of codons; SD-GC3: standard deviation of the $\mathrm{G}+\mathrm{C}$ content at the third codon position.

\section{Authors' contributions}

HS carried out the analysis and drafted the manuscript. RS and MT helped to draft the manuscript. All authors read and approved the final manuscript.

\section{Additional material}

\section{Additional file 1}

Supplemental Table S1. Statistics for the 268 bacterial genomes tested. Click here for file

[http://www.biomedcentral.com/content/supplementary/1471-

2105-10-167-S1.xls]

\section{Acknowledgements}

We thank Kazuharu Arakawa (Institute for Advanced Biosciences, Keio University) for his technical advice on the G-language Genome Analysis Environment. This research is supported by a Ministry of Education, Culture, Sports, Science and Technology of Japan Grant-in-Aid for the 2 Ist Century Centre of Excellence (COE) Program entitled 'Understanding and Control of Life via Systems Biology' (Keio University), as well as funds from the Yamagata Prefectural Government and Tsuruoka City.

\section{References}

I. Grantham R, Gautier C, Gouy M, Mercier R, Pave A: Codon catalog usage and the genome hypothesis. Nucleic Acids Res 1980, 8(I): $r 49-r 62$.

2. Sharp PM, Cowe E, Higgins DG, Shields DC, Wolfe KH, Wright F: Codon usage patterns in Escherichia coli, Bacillus subtilis, Saccharomyces cerevisiae, Schizosaccharomyces pombe, Drosophila melanogaster and Homo sapiens; a review of the considerable within-species diversity. Nucleic Acids Res 1988, I 6( I 7):8207-82 II.

3. Sueoka $\mathrm{N}$ : On the genetic basis of variation and heterogeneity of DNA base composition. Proc Natl Acad Sci USA 1962, 48:582-592.

4. Lobry JR: Asymmetric substitution patterns in the two DNA strands of bacteria. Mol Biol Evol 1996, I3(5):660-665.

5. McLean MJ, Wolfe KH, Devine KM: Base composition skews, replication orientation, and gene orientation in 12 prokaryote genomes. J Mol Evol 1998, 47(6):69I-696.

6. Sharp PM, Bailes E, Grocock RJ, Peden JF, Sockett RE: Variation in the strength of selected codon usage bias among bacteria. Nucleic Acids Res 2005, 33(4): I | 4 I- I I 53.

7. Muto $A$, Osawa $S$ : The guanine and cytosine content of genomic DNA and bacterial evolution. Proc Natl Acad Sci USA 1987, 84(I): 166-169.

8. Lafay B, Lloyd AT, McLean MJ, Devine KM, Sharp PM, Wolfe KH: Proteome composition and codon usage in spirochaetes: species-specific and DNA strand-specific mutational biases. Nucleic Acids Res 1999, 27(7): 1642-1649.

9. Mclnerney JO: Replicational and transcriptional selection on codon usage in Borrelia burgdorferi . Proc Natl Acad Sci USA 1998, 95(18): 10698-10703.

10. Ikemura T: Codon usage and tRNA content in unicellular and multicellular organisms. Mol Biol Evol 1985, 2(I): I3-34.

II. Kanaya S, Yamada Y, Kudo Y, Ikemura T: Studies of codon usage and tRNA genes of 18 unicellular organisms and quantification of Bacillus subtilis tRNAs: gene expression level and species-specific diversity of codon usage based on multivariate analysis. Gene 1999, 238(I): I43-I55.

12. Suzuki H, Saito R, Tomita M: Variation in the Correlation of G + C Composition with Synonymous Codon Usage Bias among Bacteria. EURASIP J Bioinform Syst Biol 2007:61374.

13. Grocock RJ, Sharp PM: Synonymous codon usage in Pseudomonas aeruginosa PAOI. Gene 2002, 289(I-2): $13 \mid-139$.

14. Lafay B, Atherton JC, Sharp PM: Absence of translationally selected synonymous codon usage bias in Helicobacter pylori . Microbiology 2000, I46(Pt 4):85I-860.

15. Romero $\mathrm{H}$, Zavala $\mathrm{A}$, Musto $\mathrm{H}$ : Codon usage in Chlamydia trachomatis is the result of strand-specific mutational biases and a complex pattern of selective forces. Nucleic Acids Res 2000, 28(10):2084-2090. 
16. Wright $F$ : The 'effective number of codons' used in a gene. Gene 1990, 87(I):23-29.

17. Carbone A, Zinovyev A, Kepes F: Codon adaptation index as a measure of dominating codon bias. Bioinformatics 2003, 19(16):2005-2015.

18. Sharp PM, Li WH: The codon adaptation index - a measure of directional synonymous codon usage bias, and its potential applications. Nucleic Acids Res 1987, I 5(3): I 28I- 1295.

19. Piza FF, Prado PI, Manfio GP: Investigation of bacterial diversity in Brazilian tropical estuarine sediments reveals high actinobacterial diversity. Antonie Van Leeuwenhoek 2004, 86(4):3 I7-328.

20. Watve MG, Gangal RM: Problems in Measuring Bacterial Diversity and a Possible Solution. Appl Environ Microbiol 1996, 62(II):4299-430I.

21. Wu G, Culley DE, Zhang W: Predicted highly expressed genes in the genomes of Streptomyces coelicolor and Streptomyces avermitilis and the implications for their metabolism. Microbiology 2005, I5 I (Pt 7):2 |75-2 I87.

22. Arakawa K, Tomita M: The GC skew index: a measure of genomic compositional asymmetry and the degree of replicational selection. Evol Bioinform Online 2007, 3:159-168.

23. Ermolaeva MD: Synonymous codon usage in bacteria. Curr Issues Mol Biol 2001, 3(4):91-97.

24. Karlin S: Detecting anomalous gene clusters and pathogenicity islands in diverse bacterial genomes. Trends Microbiol 200I, 9(7):335-343.

25. Lawrence JG, Ochman H: Amelioration of bacterial genomes: rates of change and exchange. I Mol Evol I997, 44(4):383-397.

26. Kerr AR, Peden JF, Sharp PM: Systematic base composition variation around the genome of Mycoplasma genitalium, but not Mycoplasma pneumoniae. Mol Microbiol 1997, 25(6): I I77-I 179.

27. Naya $\mathrm{H}$, Romero $\mathrm{H}$, Zavala $A$, Alvarez $B$, Musto $\mathrm{H}$ : Aerobiosis increases the genomic guanine plus cytosine content (GC\%) in prokaryotes. J Mol Evol 2002, 55(3):260-264.

28. Rocha EP, Danchin A: Base composition bias might result from competition for metabolic resources. Trends Genet 2002, [ 8(6):29|-294.

29. Lobry JR, Gautier C: Hydrophobicity, expressivity and aromaticity are the major trends of amino-acid usage in 999 Escherichia coli chromosome-encoded genes. Nucleic Acids Res 1994, 22(15):3174-3180.

30. Zavala $\mathrm{A}$, Naya $\mathrm{H}$, Romero $\mathrm{H}$, Musto $\mathrm{H}$ : Trends in codon and amino acid usage in Thermotoga maritima. J Mol Evol 2002 54(5):563-568.

31. Karlin S, Burge C: Dinucleotide relative abundance extremes: a genomic signature. Trends Genet 1995, I I(7):283-290.

32. Karlin S, Campbell AM, Mrazek J: Comparative DNA analysis across diverse genomes. Annu Rev Genet 1998, 32: 185-225.

33. Charif $D$, Thioulouse J, Lobry JR, Perriere G: Online synonymous codon usage analyses with the ade 4 and seqinR packages. Bioinformatics 2005, 2 I (4):545-547.

34. Suzuki $H$, Saito $R$, Tomita M: The 'weighted sum of relative entropy': a new index for synonymous codon usage bias. Gene 2004, 335:19-23.

35. Suzuki $H$, Saito $R$, Tomita M: A problem in multivariate analysis of codon usage data and a possible solution. FEBS Lett 2005 579(28):6499-6504.

36. Arakawa K, Mori K, Ikeda K, Matsuzaki T, Kobayashi Y, Tomita M: G. language Genome Analysis Environment: a workbench for nucleotide sequence data mining. Bioinformatics 2003, 19(2):305-306.

37. Arakawa K, Tomita M: G-language System as a platform for large-scale analysis of high-throughput omics data. J Pesticide Sci 2006, 3 I (3):282-288.

38. R Development Core Team: R: A language and environment for statistical computing. R Foundation for Statistical Computing, Vienna, Austria. 2007.

39. Benson DA, Karsch-Mizrachi I, Lipman DJ, Ostell J, Wheeler DL: GenBank. Nucleic Acids Res 2008:D25-30.

40. National Center for Biotechnology Information [http:// www.ncbi.nlm.nih.gov/]
Publish with Bio Med Central and every scientist can read your work free of charge

"BioMed Central will be the most significant development for disseminating the results of biomedical research in our lifetime. "

Sir Paul Nurse, Cancer Research UK

Your research papers will be:

- available free of charge to the entire biomedical community

- peer reviewed and published immediately upon acceptance

- cited in PubMed and archived on PubMed Central

- yours - you keep the copyright

Submit your manuscript here:

http://www.biomedcentral.com/info/publishing_adv.asp
BioMedcentral 